\title{
Performance of Malaria Rapid Diagnostic Test in Asymptomatic Malaria Cases in Stable Transmission Area in Kisantu, Democratic Republic of Congo
}

\author{
Gillon Ilombe ${ }^{1,2,3, *}$, Sylvie Linsuke ${ }^{2,4}$, Aimé Lulebo ${ }^{5}$, Joris Likwela ${ }^{6}$, Paulin Mutombo ${ }^{5}$, \\ Pascal Lutumba ${ }^{7}$, Jean-Pierre Van Geertruyden ${ }^{2}$, Junior Rika Matangila ${ }^{7}$ \\ ${ }^{1}$ Department of Parasitology, Unit of Entomology, National Institute of Biomedical Research, Kinshasa, Democratic Republic of Congo \\ ${ }^{2}$ Global Health Institute, University of Antwerp, Antwerp, Belgium \\ ${ }^{3}$ Unit of Clinical Pharmacology and Pharmacovigilance, University of Kinshasa, Kinshasa, Democratic Republic of Congo \\ ${ }^{4}$ Department of Epidemiology, National Institute of Biomedical Research (INRB), Kinshasa, Democratic Republic of Congo \\ ${ }^{5}$ Faculty of Medicine, School of Public Health, University of Kinshasa, Kinshasa, Democratic Republic of Congo \\ ${ }^{6}$ School of Public Health, Faculty of Medicine, University of Kisangani, Kisangani, Democratic Republic of Congo \\ ${ }^{7}$ Department of Tropical Medicine, Faculty of Medicine, University of Kinshasa, Kinshasa, Democratic Republic of Congo
}

\section{Email address:}

gillonilombe@yahoo.fr (G. Ilombe)

${ }^{*}$ Corresponding author

\section{To cite this article:}

Gillon Ilombe, Sylvie Linsuke, Aimé Lulebo, Joris Likwela, Paulin Mutombo, Pascal Lutumba, Jean-Pierre Van Geertruyden, Junior Rika Matangila. Performance of Malaria Rapid Diagnostic Test in Asymptomatic Malaria Cases in Stable Transmission Area in Kisantu, Democratic Republic of Congo. Central African Journal of Public Health. Vol. 7, No. 4, 2021, pp. 159-163. doi: 10.11648/j.cajph.20210704.12

Received: May 26, 2021; Accepted: June 11, 2021; Published: June 21, 2021

\begin{abstract}
Background: Democratic Republic of Congo has shifted from pan test to mono-specific malaria RDT tests. A new Histidine rich protein 2 (HRP2)-based Rapid Diagnostic Tests for malaria diagnosis was then implemented. Objective: This study evaluated the performances of this new diagnostic tool compared to the thick smear as the gold standard. Method: The study was conducted in Kisantu Health zone in the Democratic Republic of the Congo (DRC) where malaria is known as endemic disease. Asymptomatic children aged between 0 and 10 years were included in the study. Malaria tests such as Rapid Diagnostic test (RDT) and blood smear were perform in all enrolled children. Blood smear was considered as a reference test. In the performance analysis, only thick positive smears confirmed as $P f$ by thin smear were considered positive in calculation. The sensitivity, specificity and positive and negative predictive values of CareStart Malaria $P f$ (HRP-2) RTD Ag compared to the thick smear. Result: The prevalence of malaria infection was $78.3 \%$ and $14.7 \%$ using rapid diagnostic test and thick blood smear, respectively. Considering the gold standard, proportion of false positives was $77.5 \%$. The Sensitivity was $83.1 \%$ (CI95\%: 72.4-94.9) and specificity was $22.5 \%$ (CI95\%: 20.2-25.0). The Positive Predictive Value (PPV) was $15.2 \%$ and Negative Predictive Value (NPV) was $88.9 \%$. It appears that the HRP2-based test presently used in the Democratic Republic of Congo over estimates malaria infections. Conclusion: CareStart Malaria Pf(HRP-2) RTD Ag, currently in use in the DRC detects a very high proportion of false positives. Those children falsely positive were subject to be treated unnecessarily. Therefore, there is need of adapting the choice of RDT for malaria policy accordingly.
\end{abstract}

Keywords: Malaria, Performance, Rapid Diagnostic Test, HRP2-based Test, Kinsantu Health Zone

\section{Introduction}

The Democratic Republic of Congo (DRC) is after Nigeria, the country that bears heaviest burden of malaria [1]. To rationalize the management of malaria cases, the Artemisinin-based Combination Therapy (ACT) is restricted to patients with confirmed malaria infection [2]. In the DRC, as in many other countries, microscopy is limited to general referral hospitals, whereas the Rapid Diagnostic Test (RDT) is 
the cornerstone of malaria diagnosis in health centers and community health sites where most uncomplicated malaria cases are treated.

Since Plasmodium falciparum (Pf) is responsible for more than $90 \%$ of malaria cases in this country, malaria diagnostic policy has shifted from the use of a Pan (pLDH/ HRP-2) malaria RDT to a mono-specific test based on histidine rich protein (HRP2) [3].

The aim of this cross-sectional study was to determine the performances sensitivity, specificity as well as positive and negative predictive value of CareStart Malaria Pf (HRP-2) RTD Ag against microscopy as the gold standard, in Kinsantu $\begin{array}{llll}\text { Health Zone. } & 2 . & \text { Custom } & \text { Title }\end{array}$ (SciencePG-Level1-Multiple-line).

\section{Methods}

\subsection{Study Area}

The study was conducted in Kinsantu Health Zone, Madimba Territory, Lukaya District, Kongo Central Province, DRC. The health zone of Kisantu is constituted by 11 health areas. Malaria transmission is seasonal and many cases of malaria occur during the rainy season. In 2017, a $40 \%$ prevalence of malaria was reported by the head of the Health Zone.

\subsection{Study Design}

A cross-sectional study was conducted in Kisantu Health zone in the Democratic Republic of the Congo (DRC) where malaria is known as endemic disease. Asymptomatic children aged between 0 and 10 years were included in the study. Malaria tests such as Rapid Diagnostic test (RDT) and blood smear were perform in all enrolled children, from $1^{\text {th }}$ October to 2 November 2017, in 30 randomly selected villages located in Kisantu Health Zone.

\subsection{Study Size Calculation}

Four health Areas were randomly selected in the Health Zone. All the village in the selected Health Areas were then identified and among them, 30 villages were randomly selected to form the cluster. The number of samples to include per cluster was calculated according to the formula provided by Hayes [4], considering the incidence of malaria of $8.2 \%$ and $5.1 \%$ respectively in the control and intervention group with the ratio of $1 / 1$ between exposed and unexposed [5]. The desired power was $80 \%$ and the significance level $5 \%$. The intra-cluster correlation coefficient was 0.048 [6].

\subsection{Laboratory Procedures}

The field, the investigators collected finger prick blood samples both microscopy and RDT. All the blood slides were prepared and stained onsite, in accordance to the standard operating procedures of the study. Then, they were, all transported to the laboratory of the Tropical Medicine Department of the University of Kinshasa for reading. The complete RDT procedure for the diagnosis of malaria was carried out onsite.

\subsubsection{Blood Slide for Malaria Diagnosis}

Thick and thin blood films were prepared on the same slide for each sample collected. The slides were stained for 10 minutes, with $10 \%(1.9 \mathrm{ml})$ of fresh Giemsa working solution. Before staining, thin smears were fixed with absolute methanol [7]. Light microscopy was used for examination of the blood slides, at a magnification of $1000 x$. Hundred microscopic fields were examined in the thick smear before concluding that a blood slide was negative.

All slides were read twice by experienced microscopists. The microscopists were blinded to the RDT results. If the discrepancy was greater than $15 \%$, a third blinded reader was asked to confirm the diagnosis. The parasite density per microliter of blood was calculated using the following formula: (Number of trophozoites x 8000)/Number of leucocytes.

\subsubsection{Rapid Diagnostic Test for Malaria Infection}

Malaria infection was also diagnosed using RDT "CareStart Malaria Pf (HRP-2) RTD Ag" batch number MO17D63, manufactured by Access Bio Inc., New Jersey USA and provided free of charge by Santé Rural (SANRU). Following the manufacturer's manual, with a $5 \mu$ l capillary pipette provided, whole blood samples were collected from the finger-prick and transferred to the round sample well. Four drops of assay diluent were added to the square assay diluent well. The result was read after a minimum of 15 minutes (up to 30 minutes).

\subsection{Statistical Analysis}

The field, the investigators collected finger prick blood samples both microscopy and RDT. In the performance analysis, only thick positive smears confirmed as $P f$ by thin smear were considered positive in calculation. The sensitivity, specificity and positive and negative predictive values of CareStart Malaria Pf (HRP-2) RTD Ag compared to the thick smear considered as the "gold standard".

\section{Results}

A total of 1790 children aged to 0 to 10 years provide blood samples from 30 villages in the Health Zone of Kisantu. The number of males was 920 (51.4\%) and 51.7\% (925 children) of the study population were under five years old. None of the children had a temperature above $37.5^{\circ} \mathrm{C}$.

The prevalence of malaria infection was $78.3 \%$ and $14.7 \%$, based on malaria RDT and microscopy respectively. Furthermore, the proportion of false positives was $77.5 \%$ and proportion of false negatives was $16.9 \%$. The thin smear showed that $P f$ was predominant at $93.5 \%$ (table 1). The microscopists' agreement was high at $98 \%$ and the $2 \%$ of slides with unmatched results were re-tested by a third expert. Using thick smear as the reference test, the RDT presented the following performances: Sensibility $=83.1 \% \quad$ (CI95\%: 72.4-94.9); $\quad$ Specificity $=22.5 \% \quad$ (CI95\%: 20.2-25.0); 
$\mathrm{PPV}=15.2 \% ; \mathrm{NPV}=88.9 \%$ (see tables 2 and 3 ). The specificity decreases by $27 \%$, from $25.8 \%$ for children than under 5 years to $18.9 \%$ for children above 5 years.

When the parasitemia range was 100 to less than $1000 \mu$, the sensitivity was $81.3 \%$ (CI95\%: 65.5-99.8) and it was $86.2 \%$ (CI95\%: 72.1-100) in the range of $1000 \mu 1$ to above (Table3). The lowest parasitemia was $64 / \mu 1$ and the highest parasitemia was $61.280 / \mu 1$.

\section{Discussion}

This study aimed to evaluate the performance of CareStart Malaria Pf (HRP-2) RTD Ag compared to gold standard microscopy. We found a low specificity $(22.5 \%)$ of CareStart Malaria $P f($ HRP-2) RTD Ag while the sensitivity (83.1\%) still in acceptable range.

A number of studies, conducted in the context of stable transmission of malaria, in the DRC or other tropical region [8, $9,10,11,12]$ have reported relatively low specificity of malaria RDT, but this was never been less than $50 \%[3,14,15]$. As we recruited children living in endemic areas and tested during the rainy season, it is possible that some of them may have been successfully treated for clinical malaria during the past two or three weeks. This situation may lead to persistence of antigenemia (HRP-2 antigen) over a longer period following successful treatment and contributing to the high proportion of false positive cases reported in this study. As a limitation, the use of the molecular tool would have been important to discriminate false positives from RDTs in submicroscopic infection. It should be noted, however, that in this study, that RDT sensitivity decreased when parasite density decreased to 0 for samples with a parasite density less than $100 / \mu 1$.

Some conditions found in tropical environments, such as chronic hepatitis C, toxoplasmosis, human African trypanosomiasis, dengue, leishmaniasis, Chagas disease, and schistosomiasis and rheumatoid factor may also be considered as potential causes of false positive for malaria RDT as they can induce the production of specific IgG or IgM that may interference by binding to the capture or the signal antibody [6, $17,18,19]$. A study performed in health facilities with febrile children also highlighted poor performance of the CareStart Malaria Pf (HRP-2) RTD Ag test compared to others tests previously evaluated in DRC [20]. An RDT with such sensitivity would be suitable for malaria pre-elimination or elimination zones. In stable or high transmission areas, such very sensitive RDT will overestimate the number of confirmed malaria cases, with a potential threat of antimalarial stock outs due to increased test positivity caused by false positives cases. As a result, this could also lead to a selection of resistant strains and disorientation of health care providers to evaluate the true origin of the fever in front of a false positive [21, 22]. This can be fatal in endemic areas where malaria is generally poor and diagnostic tools are lacking.

Beside this, a proportion of $16 \%$ of false negative was observed. This may be due to the presence of a considerable prevalence of HRP-2 gene deletion [6, 23].

In 2016 a study showed that the prevalence of deletion reaching $4.2 \%$ in Kongo Central [6]. This may highlight the necessity to compare the performances of CareStart Malaria Pf (HRP-2) RTD Ag with other monospecific tests or with Pan.

The DRC has many health areas with heterogeneous malaria endemicity. In addition, the prevalence of HRP2 deletion may also be heterogeneous in the population of the health zone. Therefore, the performances and predictive values of the different tests may be useful to select the best that will fit specifics areas.

Although this has not been explicitly requested, it is possible that some children were treated for malaria in the week preceding the visit. This could explain the presence of false positives in test performance analyzes.

However, the large proportion of false positives found could not be justified by this. The prevalence of malaria in the health area of the study does not go beyond $40 \%$ according to the of the health zone report.

In conclusion, CareStart Malaria Pf (HRP-2) RTD Ag, currently in use in the DRC detects a very high proportion of false positives that can involve a considerable proportion of children treated unnecessarily. We recommend evaluating further tests to be used in the field to assess their performance especially in DRC where health areas have heterogeneous malaria endemicity. In addition, future study using the molecular test (PCR) could have been interesting to explain if the numbers of false negatives are due to presence or lack of deletion in $P f$ (HR-2). This result showed in this study conducted in Kisantu, DRC has never been reported elsewhere so far

Table 1. Performance of RDT versus TBS.

\begin{tabular}{lllll}
\hline & PPV & NPV & Sensitivity (CI95\%) & Specificity (CI95\%) \\
\hline$\geq 5$ ans & 16.6 & 92.7 & $91.5(82.2-100)$ & $18.5(10.2-20.4)$ \\
$<5$ ans & 14.9 & 86.4 & $76.1(64.9-87.3)$ & $25.8(20.6-72.3)$ \\
All & 15.7 & 88.8 & $83.7(68.8-98.5)$ & $22.5(13.2-31.7)$ \\
\hline
\end{tabular}

Table 2. Plasmodium Species Circulating in the Kisantu Health Zone.

\begin{tabular}{ll}
\hline Species & Number $(\mathbf{\%})$ \\
\hline Plasmodium falciparum & $244(93.5)$ \\
Plasmodium malariae & $10(3.83)$ \\
Plasmodium falciparum + malariae & $2(0.77)$ \\
Gametocyte plasmodium falciparum & $5(1.92)$ \\
\hline
\end{tabular}


Table 3. Sensitivity of RDT at different levels of parasitemia.

\begin{tabular}{llll}
\hline Microscopy parasitemia & $\mathbf{n}$ & RDT + & Sensitivity (CI95\%) \\
\hline ranges & & & \\
$100-1,000$ & 107 & 87 & $81.1(65,5-99,8)$ \\
$>1,000$ & 145 & 125 & $86.1(72.1-100)$ \\
\hline
\end{tabular}

\section{Acknowledgements}

The authors would like to thank all responsible of the study participants for their cooperation in providing the necessary information regarding this study, as well in providing the blood sample.

\section{References}

[1] Organization WH. World Malaria Report 2020. Geneva 2020. 2020 .

[2] Organization WH. Guidelines for the treatment of malaria; Geneva. 2010..

[3] Paludisme PNdLcl. Plan Stratégique National de Lutte contre le Paludisme 2016-2020. 2016.

[4] Gillon Ilombe VM, Hypolite Muhindo Mavoko, Raquel Inocencio da Luz, Pascal Lutumba, Jean-Pierre Van geertruyden.. Performance of HRP2-based rapid test in children attending the health center compared to asymptomatic children in the community. Malaria Journal 2014 9. 2014; 13: 308 .

[5] Swarthout TD CH, Senga RK, Van den Broek I. Paracheck-PfR accuracy and recently treated Plasmodium falciparum infections: is there a risk of over-diagnosis? Malar J 2007. 2007; 6: 58 .

[6] A-Elgayoum SME E-KA, El-Feki A, Mahgoub BA, El-Rayah E-A, Giha HA. Malaria overdiagnosis and burden of malaria misdiagnosis in the suburbs of central Sudan: special emphasis on artemisinin-based combination therapy era.. Diagnostic Microbiology and Infectious Disease 2009. 2009; 64: 20-6.

[7] Abeku TA KM, Jones C, Beard J, Mueller DH, Okia M. Determinants of the accuracy of rapid diagnostic tests in malaria case management: evidence from low and moderate transmission settings in the East African highlands. Malaria Journal 2002.7: 202.

[8] V D. Relative utility of dipsticks for diagnosis of malaria in mesoendemic area for Plasmodium falciparum and P. vivax in Northeastern India. Vector-Borne and Zoonotic Diseases 2004. 2004; 4 (2): 123-30.

[9] Mens P SN, Omar S, Heijnen M, Schallig H, Kager PA. Is molecular biology the best alternative for diagnosis of malaria to microscopy? A comparison between microscopy, antigen detection and molecular tests in rural Kenya and urban Tanzania.. Tropical Medicine and International Health 2007. 2007; 12 (2): 238-44.

[10] Hopkins H BL, Kambales W, Dokomajilar C, Rosenthal PJ, Dorsey G. Rapid diagnostic tests for malaria at sites of varying transmission intensity in Uganda. The Journal of Infectious Diseases 2008. 2008; 197: 510-8.
[11] Gerstl S DS, Mukhtar A, De Smet M, Baker A, Maikers J. Assessment of two malaria rapid diagnostic tests, with follow-up of positive pLDH test results, in a hyperendemic falciparum malaria area. Tropical Medicine and International Health 2009. 2009; 14 (Suppl 2): 92.

[12] De Oliveira AM SJ, Ouma P, Kariuki S, Barnwell J, Otieno K, et al. Malaria rapid diagnostic test use and performance by facility-based health workers in western Kenya. American Journal of Tropical Medicine and Hygiene 2007. 2007; 77: 338.

[13] De Oliveira AM SJ, Ouma PO, Kariuki S, Barnwell JW, Otieno K, et al. Performance of malaria rapid diagnostic tests as part of routine malaria case management in Kenya. American Journal of Tropical Medicine and Hygiene 2009. 2009; 80 (3): 470-4.

[14] Parr JB VR, Doctor SM, Janko M, Carey-Ewend K, Turman BJ, Keeler C, Slater HC, Whitesell AN, Mwandagalirwa K, Ghani AC, Likwela JL, Tshefu AK, Emch M, Juliano JJ, Meshnick SR.. Pfhrp2-Deleted Plasmodium falciparum Parasites in the Democratic Republic of the Congo: A National Cross-sectional Survey. J Infect Dis 2017 Jul 1. 2017; 216 (1): 36-44.

[15] Lee JH JJ, Cho CH, Kim JY, Han ET, Yun SG, et al. False-positive results for rapid diagnostic tests for malaria in patients with rheumatoid factor. Journal of clinical microbiology. 2014; 52 (10): 3784-7.

[16] Tate J WG. Interferences in immunoassay. Clin Biochem Rev 2004. 2004; 25 (2): : 105-20.

[17] Haberichter KL JP, Chittick PJ, Millward P, Robinson-Dunn B, Boyanton BL, Jr. The Brief Case: False-Positive Rapid Malaria Antigen Test Result in a Returned Traveler.. Journal of clinical microbiology. 2017; 55 (8): 2294-7.

[18] Chansuda Wongsrichanalai MJB SM, Awalludin Sutamihardja, and Walther H. Wernsdorfer. A Review of Malaria Diagnostic Tools: Microscopy and Rapid Diagnostic Test (RDT). In: Breman JG AM, White NJ, editor. Defining and Defeating the Intolerable Burden of Malaria III: Progress and Perspectives. American Journal of Tropical Medicine and Hygiene 2007; 77 (6).

[19] Hypolite Mavoko Muhindo GI, Ruth Meya, Patrick M Mitashi, Albert Kutekemeni, Didier Gasigwa, Pascal Lutumba, Jean-Pierre Van Geertruyden. Accuracy of malaria rapid diagnosis test Optimal-IT ${ }^{\circledR}$ in Kinshasa, the Democratic Republic of Congo. Malar J 2012. 2012; 11: 224.

[20] Koram KA MM. When is "malaria" malaria? The different burdens of malaria infection, malaria disease, and malaria-like illnesses. The American journal of tropical medicine and hygiene. 2007; 77 (6 Suppl): 1-5.

[21] Bell DR WD, Martin LB. False-positive results of a Plasmodium falciparum histidine-rich protein 2-detecting malaria rapid diagnostic test due to high sensitivity in a community with fluctuating low parasite density.. The American journal of tropical medicine and hygiene. 2005; 73 (1): 199-203. 
[22] Wellems TE W-JA, Panton LJ. Genetic mapping of the chloroquine-resistance locus on Plasmodium falciparum chromosome 7.. Proceedings of the National Academy of Sciences of the United States of America 1991; 88 (8): 3382-6.
[23] Biswas S TD, Rao DN.. Investigation of the kinetics of histidine-rich protein 2 and of the antibody responses to this antigen, in a group of malaria patients from India. Annals of tropical medicine and parasitology. 2005; 99 (6): 553-62. 